\title{
A influência da sustentabilidade sobre o desempenho exportador no mercado internacional da carne bovina
}

\author{
The influence of sustainability on the export \\ performance in the beef international market
}

\author{
Francisco Sperotto Flores ${ }^{1}$ \\ Iuri Gavronski²
}

\section{Resumo}

O objetivo deste trabalho é avaliar o relacionamento entre a sustentabilidade e o desempenho exportador dos frigoríficos exportadores de carne bovina. Para isso, foi utilizada análise de dados em painel a partir da coleta de dados secundários das exportações de carne bovina in natura fresca ou resfriada, oriundas de diversos países, para os mercados italiano, norte-americano, holandês, francês, mexicano, japonês, alemão e para o Reino Unido, entre os anos de 2002 e 2012, resultando em uma base com 959 observações. A sustentabilidade dos exportadores foi avaliada por meio do seu desempenho social, utilizando como proxy o Índice de Desenvolvimento Humano (IDH), e do desempenho ambiental, avaliado a partir da Poupança Líquida Ajustada (ANS). Os resultados obtidos pela análise econométrica demonstram que a dimensão social da sustentabilidade exerce uma influencia positiva sobre o desempenho exportador dos frigoríficos exportadores de carne bovina, enquanto a dimensão ambiental da sustentabilidade está associada à capacidade de entrada e manutenção destas empresas no mercado internacional da carne bovina.

Palavras-chave: Desempenho exportador. Sustentabilidade. Desempenho social. Desempenho ambiental.

\section{Abstract}

The purpose of this study is evaluate the relationship between sustainability and the export performance of beef exporters. It is used panel data analysis from the

Mestre em Administração - UNISINOS Assistente em Administração - IF Farroupilha Campus Santo Augusto - Brasil - E-mail: francisco.flores@iffarroupilha.edu.br

2 Doutor em Administração pelo PPGA/UFRGS, Professor do Programa de Pós-Graduação em Administração - UNISINOS - Brasil - E-mail: igavronski@unisinos.br 
collection of secondary data of exports of in natura fresh or chilled beef with regard to Italy, Netherlands, French, Mexico, Japan, German and UK between the years 2002 and 2012, resulting in a database with 959 observations. The sustainability of exporters was evaluated through its social performance, using as proxy the Human Development Index (HDI), and environmental performance, evaluated from the Net Adjusted Savings (ANS). The results obtained by econometric analysis show that the social dimension of sustainability has a positive influence on the export performance of beef exporters, while the environmental dimension of sustainability is associated with capacity of these companies access and maintenance in the international beef market.

Keywords: Export performance. Sustainability. Social performance. Environmental performance.

\section{Introdução}

Acompanhando a intensificação do comércio internacional após a Segunda Guerra Mundial, as exportações de carne bovina no mercado mundial passaram de cerca de US\$ 800 milhões em 1961, com um rebanho de 950 milhões de cabeças de gado, para US\$ 39 bilhões em 2011, com um rebanho de cerca de 1,5 bilhão de cabeças (FAO, 2014). Entretanto, a produção agropecuária bovina tem um grande impacto sobre os desequilíbrios causados pelas atividades produtivas ao meio ambiente e à sociedade, sendo responsável por $18 \%$ das emissões de gases do efeito estufa; além disso, $34 \%$ das terras cultivadas no mundo são destinadas para a produção de ração animal, além de ocupar $80 \%$ da área agrícola global (STEHFEST et al., 2013). A má utilização de nutrientes no solo (nitrogênio, fósforo e potássio) é responsável pela contaminação de recursos hídricos, enquanto a abertura de novas áreas de pastagens é responsável pela destruição de ecossistemas, por meio do desmatamento e do uso inadequado do solo (ZEN et al., 2008).

Do ponto de vista social, a expansão da produção pecuária bovina é responsável pelo deslocamento da população rural para outras regiões, visto que ela tem uma capacidade menor de geração de empregos em comparação com outras atividades agropecuárias. Ao mesmo tempo, é uma das atividades produtivas em que há maior número de registros da 
utilização de trabalho escravo, principalmente em regiões de expansão da fronteira agrícola, caracterizadas por terem baixo desenvolvimento econômico e social (SCHLESINGER, 2010).

Os efeitos colaterais gerados pelo crescimento da produção agropecuária ajudaram a aprofundar desigualdades econômicas e sociais, devido à crescente concentração de terra e renda, e a agravar problemas ambientais, como o desmatamento e a contaminação do solo e da água. Diante desse cenário, o setor se tornou suscetível ao aumento do controle regulatório de governos e organizações internacionais, danos à imagem e, principalmente, riscos financeiros devido ao mau uso de recursos naturais pelas atividades produtivas.

Este estudo pretende responder ao seguinte questionamento: qual a influência da sustentabilidade sobre o desempenho das exportações de carne bovina? Para isso, será analisada a relação entre sustentabilidade, a partir das suas dimensões social e ambiental, e o desempenho dos principais exportadores de carne bovina. A partir do crescimento de problemas socioambientais relacionados às atividades produtivas, na segunda metade do século $X X$, emerge a necessidade de uma nova postura ética, baseada na responsabilidade da sociedade atual pela qualidade de vida e as condições de sobrevivência das gerações futuras, exigindo que governos e empresas adotem uma postura condizente com os ideais do desenvolvimento sustentável. Como consequência, as empresas passaram a ser responsabilizadas pelos impactos gerados por suas atividades produtivas, incluindo os efeitos das operações dos seus fornecedores e toda a cadeia de suprimentos, exigindo que elas se adéquem às demandas da sociedade local e do mercado externo.

Frente às exigências dos mercados consumidores, o setor produtivo da carne bovina passou a se adaptar a uma série de exigências e regulamentações impostas pelos países importadores, as quais levaram ao aprimoramento dos sistemas de gestão e dos processos produtivos. Essas medidas impulsionaram os países produtores a adotar práticas de controle sanitário e de rastreabilidade da produção, melhores práticas de manejo do rebanho e medidas de redução do impacto social 
e ambiental das suas atividades (MALAFAIA et al., 2010), impulsionando o surgimento de iniciativas que buscam disseminar princípios e práticas que contribuam para o desenvolvimento de uma pecuária sustentável, como o Grupo de Trabalho da Pecuária Sustentável (GTPS), no Brasil, e o Sustainable Food Exports Group, na Nova Zelândia.

Embora essas iniciativas contribuam para a discussão sobre o papel da sustentabilidade dentro do setor produtivo da carne bovina, são pontuais, tendo impacto apenas local, mostrando ainda poucas evidências do impacto sobre a competitividade das empresas que se engajam nessas iniciativas e o desempenho das exportações de carne bovina.

\section{Modelo de pesquisa}

A Figura 1 expõe o modelo teórico proposto para o relacionamento entre sustentabilidade e desempenho exportador. A sustentabilidade é avaliada a partir das suas dimensões ambiental e social. $O$ desempenho exportador é definido como o resultado das operações de exportação. Nas seções a seguir, são apresentados os pressupostos teóricos que dão suporte ao modelo.

Figura 1: Modelo teórico

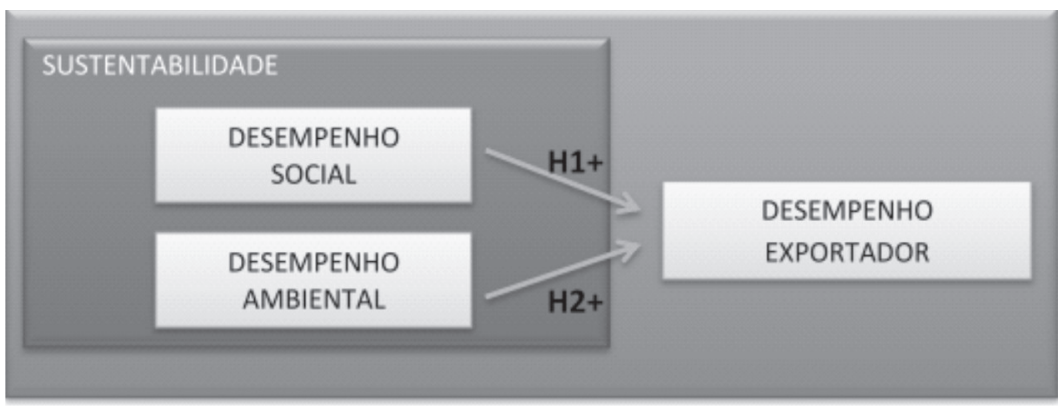

Fonte: Elaborado pelos autores. 


\subsection{Desempenho exportador}

Os estudos que examinam os fatores associados ao sucesso das exportações refletem uma preocupação com as políticas macro, relacionadas aos acordos internacionais e as políticas de exportação, além de um foco micro, preocupado com a competitividade das empresas no mercado exportador (ZOU; TAYLOR; OSLAND, 1998). Entretanto, apesar de o desempenho exportador ser um dos temas mais pesquisados dentro dessa área de pesquisa, continua sendo um dos temas mais controversos e mal compreendidos da área, devido à grande variedade de indicadores e determinantes utilizados para medir o construto, gerando inconsistências que dificultam a comparação e interpretação dos resultados obtidos por esses estudos (CARNEIRO; ROCHA; SILVA, 2011).

A Resource Based View (RBV) contribui para esse debate ao propor que o desempenho organizacional é resultado do conjunto de recursos e capacidades das firmas, os quais, articulados, formam as competências essenciais das empresas (PRAHALAD; HAMEL, 1990). De acordo com essa corrente teórica, o desempenho da firma é determinado por características estruturais dos mercados, que definem a intensidade competitiva que a empresa enfrenta e, também, a capacidade de a empresa obter e manter vantagens competitivas pela execução das suas estratégias competitivas (MORGAN; KALEKA; KATSIKEAS, 2004). Os recursos exclusivos da firma e sua capacidade organizacional são tidos como as principais fontes de vantagens competitivas (PRAHALAD; HAMEL, 1990), o que inspirou uma série de estudos que consideram recursos e capacidades da firma, como o tamanho, a experiência internacional e a orientação para o mercado, como determinantes do desempenho exportador. Assim, os fatores internos da firma são apontados como um dos principais fatores para o início, desenvolvimento, manutenção e sucesso das operações de exportação de uma empresa (LEONIDOU; KATSIKEAS; PIERCY, 1998).

Embora os fatores internos sejam fundamentais para o sucesso empresarial, as empresas continuam dependentes das suas interações 
com o ambiente externo. Nesse sentido, o paradigma contingencial apresenta argumentos complementares à RBV acerca dos determinantes de desempenho exportador, ao propor que o desempenho organizacional é dependente da adequação da estratégia e da estrutura organizacional ao ambiente em que a firma está inserida (CHANDLER, 1969; LAWRENCE; LORSCH, 1967). As organizações dependem do seu ambiente para obter recursos e devem gerenciar essa dependência por meio do desenvolvimento e manutenção de estratégias que permitam o acesso a estes recursos (SOUSA; MARTÍNEZ-LÓPEZ; COELHO, 2008). Nesse contexto, os fatores externos e a estratégia de exportação da firma são os principais determinantes do desempenho exportador, podendo ser classificados em características do mercado externo e características do mercado doméstico.

Ainda que a literatura tenha listado uma série de fatores determinantes, a necessidade de um melhor entendimento sobre a influência destes e mudanças no ambiente econômico, político e social alteram as condições para que as empresas acessem recursos e capacidades que lhes garantam vantagens competitivas no mercado internacional, abrindo a possibilidade para investigações sobre novos fatores determinantes de desempenho exportador.

Diante da crescente preocupação da sociedade com o impacto das atividades econômicas sobre o meio ambiente e as pessoas, a interação entre questões socioambientais e comerciais se intensificaram, com os consumidores cada vez mais conscientes sobre as consequências dos seus atos de consumo, pressionando governos e empresas a se adaptarem às demandas da sociedade local e do mercado externo, buscando reduzir os riscos associados a problemas socioambientais (ANDERSEN; SKJOETT-LARSEN, 2009; LEONIDOU et al., 2013). Entretanto, a literatura vem relegando esse tema, havendo ainda poucos estudos que buscam encontrar uma ligação entre desempenho exportador e desempenho sustentável (GALDEANO-GÓMEZ, 2010; MARTÍN-TAPIA; ARAGÓN-CORREA; RUEDA-MANZANARES, 2010). A seguir, é apresentado o conceito de sustentabilidade, seguido por uma discussão do seu impacto sobre o desempenho exportador. 


\subsection{Sustentabilidade}

A concepção de sustentabilidade utilizada por este estudo é derivada do conceito de desenvolvimento sustentável proposto em 1987 pela Comissão de Desenvolvimento Sustentável das Nações Unidas (WCED), através do relatório Our Common Future, definido como o desenvolvimento que "atende às necessidades do presente sem comprometer a capacidade de as gerações futuras atenderem suas próprias necessidades" (WCED, 1987, p. 8). Entretanto, mesmo sendo utilizado de forma ampla, o conceito ainda está longe de assumir um significado consensual, sendo analisado e caracterizado sob diferentes dimensões e aspectos (CARTER; ROGERS, 2008; HART, 1995).

Buscando operacionalizar o conceito em um contexto empresarial, John Elkington (1997) propõe o conceito de triple bottomline, buscando equilibrar objetivos econômicos, sociais e ambientais, considerando que as atividades produtivas afetam a sociedade e o meio ambiente, ao mesmo tempo em que podem gerar vantagens competitivas e benefícios econômicos para as empresas (CARTER; ROGERS, 2008). Nesse sentido, uma empresa sustentável é aquela que consegue, ao mesmo tempo, gerar lucro aos acionistas, melhorar a qualidade de vida das pessoas com quem interage e proteger o meio ambiente, conciliando os interesses do negócio, da sociedade e do meio ambiente (LEE; KIM, 2009).

A dimensão econômica da sustentabilidade busca garantir que os acionistas obtenham a liquidez e o retorno financeiro desejado sobre o capital investido (DYLLICK; HOCKERTS, 2002). Friedman (1970) considera que, embora as questões ambientais e sociais sejam muito importantes, são incompatíveis com o objetivo organizacional de gerar lucro. Segundo essa visão, o papel social da empresa restringe-se a gerar empregos, pagar salários justos e manter seus impostos em dia, enquanto as ações de responsabilidade social e ambiental gerariam custos adicionais, consequentemente, reduzindo a rentabilidade da firma (DIXON-FOWLER et al., 2012; HULL; ROTHENBERG, 2008; KING; LENOX, 2002). 
Por outro lado, diversos autores argumentam que a empresa que leva em consideração apenas seus objetivos financeiros, em detrimento das dimensões ambiental e social, está comprometendo sua capacidade em longo prazo (DYLLICK; HOCKERTS, 2002; PORTER; KRAMER, 2006). Para os autores que defendem uma postura empresarial sustentável, as empresas que possuem um desempenho social e ambiental superior são capazes de atrair e reter funcionários qualificados, reduzir custos de produção e melhorar a eficiência operacional, além de criar oportunidades de mercado que fazem com que estas empresas tenham um desempenho econômico superior (AMEER; OTHMAN, 2012; GAVRONSKI; FERRER; PAIVA, 2008; HULL; ROTHENBERG, 2008). Para aprofundar a análise, as próximas seções buscam discutir como o desempenho socioambiental das empresas pode influenciar seu desempenho exportador.

\subsection{Desempenho social}

Adimensão social da sustentabilidade busca reduzir a desigualdade entre padrões de vida, promovendo igualdade de acesso a recursos e serviços mediante a distribuição igualitária de renda e bens (MARTINS; OLIVEIRA, 2005), da criação de valor para comunidades locais por meio do aumento do capital humano e da promoção do capital social (DYLLICK; HOCKERTS, 2002). Bowen (1953) e Carrol (1979) argumentam que, além de perseguir seus objetivos econômicos, as empresas têm a obrigação de atingir os mais altos padrões éticos e sociais, atendendo expectativas econômicas, éticas, legais e discricionárias que a sociedade tem sobre elas. Essas expectativas geram uma série de pressões externas, lideradas pela mídia, consumidores e parceiros comerciais, tornando recorrente a exigência para que as empresas comprovem que seus processos cumprem requisitos éticos e legais. As empresas que têm um desempenho social abaixo do esperado, avaliado pelos requisitos éticos e legais impostos pelo seu ambiente institucional, correm o risco de sofrer sanções econômicas e comerciais, podendo ser excluídas do mercado se, de alguma forma, gerarem impactos negativos às comunidades onde atuam ou operarem de forma socialmente irresponsável, não cumprindo 
com suas obrigações trabalhistas ou utilizando-se de mão de obra infantil ou trabalho escravo, forçado ou degradante.

Buscando se antecipar às pressões do ambiente institucional pela adoção de práticas sustentáveis, uma série de empresas vem agindo de forma proativa, de modo a obter um desempenho social superior, conseguindo capturar valor para a firma. Por meio do desenvolvimento de estratégias sustentáveis, essas empresas são capazes de obter vantagens competitivas através da criação de uma reputação socialmente responsável, possibilitando a cobrança de valores superiores por seus produtos e o acesso aos principais mercados consumidores (BOEHE; CRUZ, 2010). Ao mesmo tempo em a reputação dessas empresas Ihes garante vantagens mercadológicas, facilita o recrutamento de pessoal qualificado, o que possibilita aumentar sua eficiência e elevar sua lucratividade (RUSSO; FOUTS, 1997). Palma et al. (2014) apresentam evidências de que empresas que buscam facilitar o acesso a produtos e serviços, proporcionando um melhor nível de vida aos seus stakeholders, principalmente no mundo em desenvolvimento, visualizam ganhos de produtividade e de imagem corporativa que potencializam sua participação no mercado, possibilitando criar uma plataforma de entrada a novos mercados de exportação e melhorar seu desempenho exportador.

Embora o mercado internacional ainda considere preço e qualidade como os principais requisitos na seleção de fornecedores, diante das pressões exercidas pelos stakeholders para que as empresas atinjam um desempenho social superior, a adoção de práticas socialmente sustentáveis vem sendo uma característica que ganha importância nas decisões de compras, principalmente em mercados desenvolvidos (BELLESI; LEHRER; TAL, 2005). Neles, empresas com um desempenho social superior têm obtido um desempenho exportador superior, através de eventuais reduções de custos resultantes da adoção de processos produtivos mais eficientes, do aumento do volume de vendas, além de cobrarem preços relativamente mais altos frente a seus concorrentes com um desempenho social inferior (LEONIDOU et. al., 2013; MATUTE; 
FRAJ; MARTI, 2011). Nesse sentido, é proposta a Hipótese 1 da pesquisa:

Hipótese 1: O desempenho social está positivamente relacionado com o desempenho exportador dos frigoríficos exportadores de carne bovina.

\subsection{Desempenho ambiental}

À medida que as pressões sociais para que as empresas passem a atuar de forma responsável evoluem, o principal desafio passa a ser equilibrar os objetivos econômicos e sociais das organizações com a redução dos impactos ambientais de suas atividades produtivas (ORLITZKY; SIEGEL; WALDMAN, 2011). A dimensão ambiental da sustentabilidade está associada à utilização racional dos recursos naturais disponíveis, tendo por objetivo principal a redução do impacto das atividades produtivas sobre o ecossistema, através do desenvolvimento de processos mais eficientes, da redução de desperdício de materiais e da prevenção de incidentes ambientais (DIXON-FOWLER et al., 2012; KING; LENOX, 2002).

Galdeano-Gómez (2010) avalia que o crescimento da atividade econômica, resultante da intensificação do comércio internacional, pressiona as empresas a buscar formas de reduzir custos e aumentar sua eficiência produtiva. As pressões competitivas criam incentivos para que as empresas ajam de forma oportunista (KING; LENOX, 2002), transferindo seus processos produtivos que geram passivos socioambientais para outras empresas ou outros países com níveis de desenvolvimento social mais baixo, ou para onde houver menos controle quanto ao impacto das atividades produtivas sobre o meio ambiente (WHEELER, 2001).

O ambiente externo impõe pressões a que as empresas respondem por meio do desenvolvimento e implementação de estratégias sustentáveis que podem levar a ganhos de competitividade em relação a empresas que têm um desempenho ambiental inferior, através do 
desenvolvimento de processos produtivos mais eficientes que permitem um melhor aproveitamento dos recursos produtivos disponíveis, ao mesmo tempo em que reduzem os riscos e os custos associados à emissão de poluentes por suas atividades produtivas (MARTÍN-TAPIA; ARAGÓN-CORREA; RUEDA-MANZANARES, 2010; PORTER; LINDE, 1995). Embora as dificuldades impostas pelo mercado criem incentivos para que as empresas atuem de forma ambientalmente irresponsável, a adoção de práticas ambientalmente responsáveis e a subsequente certificação delas possibilita às cadeias produtivas reduzir assimetrias de informação e o risco de comportamento oportunista pelos agentes da cadeia, levando as empresas a obter vantagens competitivas e um desempenho exportador superior (HART, 1995; LEONIDOU et. al. 2013; RODRÍGUEZ-RODRIGUEZ et al, 2012).

Evidências das vantagens obtidas através da adoção de estratégias ambientais sustentáveis são apresentadas por Antonietti e Marzucchi (2013), os quais constataram que empresas com um desempenho ambiental superior obtêm um aumento de eficiência produtiva que resulta em uma redução dos custos operacionais e aumento de receitas. Os investimentos no desenvolvimento de processos sustentáveis permitem um aumento da eficiência produtiva que resulta na redução de custos operacionais, através do melhor aproveitamento de matérias-primas, uso de energia e bens de capital, melhorando a imagem corporativa e permitindo reduzir custos associados a litígios e multas decorrentes de problemas ambientais (MARTÍN-TAPIA; ARAGÓN-CORREA; SENIZEBARRIO 2008; PALMA et al., 2014). Ao mesmo tempo em que permite uma redução de custos operacionais, empresas com um desempenho ambiental superior conseguem obter acesso facilitado a mercados com normas ambientais mais rigorosas (VIEIRA; TRAILL, 2008), possibilitando um aumento das receitas de venda e, consequentemente, desempenho exportador e desempenho econômico superior ao obtido por empresas com padrões ambientais inferiores. Dessa forma, as vantagens auferidas garantem a possibilidade de maximizar seu desempenho exportador, devido à maior aceitação dos seus produtos, principalmente em países desenvolvidos, por atender a um padrão de produção mais elevado e 
facilitar a entrada dos seus produtos nestes mercados considerados de difícil acesso. Nesse sentido, é proposta a segunda hipótese da pesquisa.

Hipótese 2: 0 desempenho ambiental está positivamente relacionado com o desempenho exportador dos frigoríficos exportadores de carne bovina.

\section{Materiais e métodos}

\subsection{Amostra}

O objetivo deste estudo é avaliar o relacionamento entre a sustentabilidade e o desempenho das exportações de carne bovina. Para isso, foi utilizada a análise de dados em painel, a partir da coleta de dados secundários das exportações de carne bovina in natura fresca ou resfriada, oriundas de diversos países, para Itália, EUA, Holanda, França, México, Japão, Alemanha e Reino Unido, entre os anos de 2002 e 2012. Esses mercados foram responsáveis por $70 \%$ das importações de carne bovina in natura fresca ou resfriada durante o período da análise. Nesses países, a maior parte do produto na forma in natura fresca ou resfriada importada busca atender o consumidor final, sem passar por novas fases de processamento, podendo ser mais afetada pelos requisitos de sustentabilidade do que outras commodities agrícolas, como a carne congelada ou enlatada, milho e soja. Foram considerados exportadores os países que realizaram embarques não inferiores à capacidade máxima de um contêiner, 18.000 quilos (ISO, 2014), durante pelo menos cinco anos consecutivos. Foram desconsideradas as informações referentes aos países que atuam como plataforma de entrada para produtos alimentícios em outros mercados, onde o volume exportado de produtos cárneos excede o volume produzido.

A utilização de dados nacionais se deve à escassez de informações no âmbito da firma, o que é referendado por estudos como os de Dyck e Nelson (2003) e Flores (2012). Entretanto, considerando-se que o mercado internacional da commodity é dominado por poucas empresas 
multinacionais, visto que $50 \%$ das exportações de carne bovina são feitas por empresas brasileiras, que lideram o processamento do produto em países como Argentina e Uruguai, $60 \%$ da produção australiana é destinada à exportação, $50 \%$ da produção total do país pertence a firmas de propriedade estrangeira ou joint ventures com firmas estrangeiras, e quatro empresas concentram $85 \%$ do processamento de carne bovina nos Estados Unidos, os dados nacionais refletem o comportamento das firmas no mercado internacional.

\subsection{Variáveis da pesquisa}

A variável dependente do presente estudo é o desempenho exportador dos países exportadores de carne bovina nos mercados importadores, enquanto as variáveis dependentes do estudo são o desempenho social e o desempenho ambiental desses países. As informações utilizadas na pesquisa contemplam o período entre os anos de 2002 a 2012, período caracterizado por um forte crescimento do consumo mundial de carne bovina, acompanhado do crescimento de $11 \%$ da produção de carne bovina a nível mundial (USDA, 2014), que pode ter resultar em um impacto significativo sobre o meio ambiente e a sociedade. Dessa forma, a análise desse período é fundamental para o entendimento do comportamento do mercado internacional da carne bovina frente às pressões institucionais para a redução do impacto das suas atividades produtivas sobre o meio ambiente e a sociedade.

As informações sobre o desempenho exportador foram obtidas na base de dados COMTRADE (2014), base estatística da ONU que registra o comércio mundial de commodities. Para mensurar o desempenho exportador, foi utilizado como variável o volume transacionado de carne fresca ou resfriada registrado na base, devido à alta correlação entre o volume e o valor transacionado do produto. Para reduzir a amplitude dos valores da variável e a sensibilidade das estimativas a valores extremos, foi utilizado o logaritmo do volume importado, procedimento recomendado por Wooldridge (2013). 
Para avaliar o desempenho social, foi utilizado como proxy de desempenho social o Índice de Desenvolvimento Humano (IDH) dos países exportadores. O índice, que é publicado pela ONU anualmente, desde sua criação em 1990, é uma das medidas mais reconhecidas de desenvolvimento social, baseado em uma lógica na qual o desenvolvimento humano elevado facilita o desenvolvimento sustentável. As informações para a análise foram obtidas através da ONU (2014b), por meio da base de dados UNdata.

Para mensurar o desempenho ambiental, foi utilizado como proxy a Poupança Líquida Ajustada (ANS). Indicador baseado nos conceitos de contas nacionais verdes, calculado pelo Banco Mundial, busca medir a taxa de crescimento de uma economia levando em conta os investimentos em capital humano, o esgotamento dos recursos naturais e danos causados pela poluição (BANCO MUNDIAL, 2014). O conjunto de dados utilizado pela pesquisa foi obtido através da base de dados World Data Bank do Banco Mundial (2014b).

\subsection{Técnicas de análise de dados}

Para identificar as relações existentes entre as variáveis da pesquisa, foram realizadas análises estatísticas dos dados obtidos pelo estudo, através do software StataSE 13 (STATACORP, 2013). Em um primeiro momento, foi realizado um procedimento para a identificação de outliers dentro das variáveis do estudo. Outliers são observações que apresentam valores distantes dos demais valores de uma série estatística, exercendo uma influência desproporcional no relacionamento entre as variáveis (AGUINIS; GOTTFREDSON; JOO, 2013). Os autores propõem a existência de diferentes formas de valores extremos, classificados em Interesting Outliers, Influential Outliers e Error Outliers. Error Outliers são valores extremos resultados de erros no procedimento de amostragem, podendo ser identificados através da combinação de ferramentas visuais, como gráficos e diagramas, e ferramentas de análise quantitativas, como a análise do desvio padrão. Desse modo, foi utilizado o algoritmo de Bacon, proposto por Billor, Hadi e Velleman (2000), seguido da análise 
visual das observações apontadas por esta análise como discrepantes. Após a identificação desses casos, foram consultadas as bases de dados, das quais foram coletadas as informações utilizadas na pesquisa, verificando-se que os valores apontados como discrepantes pareciam ser provenientes de informações imprecisas nas bases de dados, sendo assim excluídos das análises subsequentes, diante da impossibilidade de se adotar procedimentos de correção desses valores.

A fim de verificar se a forma mais adequada de fazer as estimações seria por meio de um modelo de regressão de efeitos fixos ou aleatórios, foi realizado o teste de Hausman. Para avaliar o comportamento do modelo proposto e a confiabilidade dos seus resultados, foi calculado o fator de inflação da variância (FIV), para verificar a presença de multicolinearidade entre as variáveis do estudo. Para testar a homocedasticidade nos resíduos, foi feito o teste de Breush-Pagan-Godfrey e, para verificar a presença de autocorrelação entre os resíduos, o teste de Wooldridge.

\subsection{Modelo econométrico}

Tendo em vista que o conjunto de dados obtido consiste em uma série temporal para cada registro obtido, foi utilizada uma estrutura de dados em painel para análise das informações coletadas. Um conjunto de dados em painel consiste em uma série temporal para cada registro de um corte transversal de uma série de dados, acompanhando as mesmas unidades do corte transversal ao longo do tempo, permitindo controlar características não observáveis dos indivíduos e estudar a importância das defasagens do comportamento desses indivíduos, ou o resultado de tomar decisões (WOOLDRIDGE, 2013). Dessa forma, a especificação do modelo econométrico geral utilizado para a análise consiste em:

$$
\operatorname{Dexp}_{\mathrm{ijt}}=\beta_{0}+\operatorname{Dso}_{\mathrm{it}} \beta_{1}+\operatorname{Dam}_{\mathrm{it}} \beta_{2}+\alpha_{\mathrm{i}}+\mu_{\mathrm{it}}
$$

Em que $D \exp _{i j t}$ representa as exportações de carne fresca ou resfriada do país $i$ para o país $j$ no ano $t ; D s o_{i t} \beta_{1}$, o desempenho social país $i$ no ano $t ; \operatorname{Dam}_{i t} \beta_{2}$, o desempenho ambiental do país $i$ no ano $t ; \alpha_{i}$ representa um componente do erro que varia com $i$, mas permanece 
constante ao longo do tempo, podendo estar correlacionada com as variáveis explicativas; $\mu_{\mathrm{it}}$ refere-se ao componente de erro que varia em função de $i$ e $t$.

\section{Resultados}

A presente seção apresenta o resultado das estimações realizadas a partir do conjunto de dados obtido dos importadores de carne in natura fresca ou resfriada. Em um primeiro momento, são apresentadas as estatísticas descritivas da amostra, seguida dos testes de especificação e da análise do modelo econométrico.

\subsection{Análise descritiva}

A análise descritiva das amostras e o resultado dos testes de multicolinearidade são apresentados na Tabela 1. A exclusão dos valores extremos e referentes aos países onde o volume exportado de produtos cárneos excede o volume produzido resultou em um painel não balanceado, com 959 observações.

Em média, os países da amostra transacionaram cerca de 17.500 toneladas de carne bovina fresca ou resfriada com cada parceiro comercial. Responsáveis por aproximadamente 32\% das importações mundiais de carne bovina in natura fresca ou resfriada, Itália, França e Estados Unidos têm os parceiros que atingiram melhor desempenho médio do indicador de desempenho ambiental, com ANS de 11,16\%, $11,22 \%$ e $10,20 \%$, respectivamente, enquanto o desempenho ambiental médio dos exportadores durante o período analisado foi de $9,32 \%$.

Embora os importadores tenham efetuado transações com países com desenvolvimento humano baixo e médio, conforme pode ser verificado nos valores mínimos da variável desempenho social, o IDH médio dos países exportadores (0.861) indica que as importações de carne bovina in natura fresca ou resfriada dos países analisados são feitas preferencialmente de países com desenvolvimento humano elevado ou muito elevado. 
Tabela 1 - Análise descritiva das amostras.

\begin{tabular}{|c|c|c|c|c|c|c|}
\hline Modelo & Variável & Obs. & Média & $\begin{array}{l}\text { Desvio } \\
\text { Padrão }\end{array}$ & Mínimo & Máximo \\
\hline Itália & $\begin{array}{l}\text { Dexp (Log) } \\
\text { Dam } \\
\text { Dso }\end{array}$ & $\begin{array}{l}119 \\
119 \\
119\end{array}$ & $\begin{array}{c}16,2345 \\
11,16 \\
0,894\end{array}$ & $\begin{array}{c}1,869762 \\
3,87 \\
0,022\end{array}$ & $\begin{array}{c}10,39038 \\
1,78 \\
0,850\end{array}$ & $\begin{array}{c}18,34628 \\
20,94 \\
0,938\end{array}$ \\
\hline EUA & $\begin{array}{l}\text { Dexp (Log) } \\
\text { Dam } \\
\text { Dso }\end{array}$ & $\begin{array}{l}89 \\
89 \\
89\end{array}$ & $\begin{array}{c}15,4997 \\
10,20 \\
0,791\end{array}$ & $\begin{array}{c}2,241989 \\
3,55 \\
0,123\end{array}$ & $\begin{array}{c}9,854875 \\
2,65 \\
0,549\end{array}$ & $\begin{array}{c}19,7 \\
18,80 \\
0,938\end{array}$ \\
\hline Holanda & $\begin{array}{l}\text { Dexp (Log) } \\
\text { Dam } \\
\text { Dso }\end{array}$ & $\begin{array}{l}201 \\
201 \\
201\end{array}$ & $\begin{array}{c}14,83527 \\
9,26 \\
0,865\end{array}$ & $\begin{array}{c}2,520957 \\
5,09 \\
0,040\end{array}$ & $\begin{array}{c}9,846917 \\
-3,14 \\
0,761 \\
\end{array}$ & $\begin{array}{c}18,06087 \\
24,18 \\
0,938\end{array}$ \\
\hline França & $\begin{array}{l}\text { Dexp (Log) } \\
\text { Dam } \\
\text { Dso }\end{array}$ & $\begin{array}{l}159 \\
159 \\
159 \\
\end{array}$ & $\begin{array}{c}14,85639 \\
10,22 \\
0,879\end{array}$ & $\begin{array}{c}2,520957 \\
5,09 \\
0,040\end{array}$ & $\begin{array}{c}9,846917 \\
-3,14 \\
0,761\end{array}$ & $\begin{array}{c}18,06087 \\
24,17 \\
0,938\end{array}$ \\
\hline México & $\begin{array}{l}\text { Dexp (Log) } \\
\text { Dam } \\
\text { Dso }\end{array}$ & $\begin{array}{l}49 \\
49 \\
49\end{array}$ & $\begin{array}{c}14,90731 \\
7,85 \\
0,824 \\
\end{array}$ & $\begin{array}{c}3,271182 \\
5,13 \\
0,128 \\
\end{array}$ & $\begin{array}{c}9,805489 \\
-4,14 \\
0,549 \\
\end{array}$ & $\begin{array}{c}19,44596 \\
18,80 \\
0,938 \\
\end{array}$ \\
\hline Japão & $\begin{array}{l}\text { Dexp (Log) } \\
\text { Dam } \\
\text { Dso }\end{array}$ & $\begin{array}{l}50 \\
50 \\
50\end{array}$ & $\begin{array}{c}16,13095 \\
8,25 \\
0,889\end{array}$ & $\begin{array}{c}2,02031 \\
2,73 \\
0,062\end{array}$ & $\begin{array}{c}10,64039 \\
2,60 \\
0,745\end{array}$ & $\begin{array}{c}19,2222 \\
13,37 \\
0,938\end{array}$ \\
\hline Alemanha & $\begin{array}{l}\text { Dexp (Log) } \\
\text { Dam } \\
\text { Dso }\end{array}$ & $\begin{array}{l}189 \\
189 \\
189\end{array}$ & $\begin{array}{c}14,2939 \\
9,23 \\
0,870\end{array}$ & $\begin{array}{c}2,216539 \\
4,04 \\
0,046\end{array}$ & $\begin{array}{c}9,841612 \\
-0,17 \\
0,742\end{array}$ & $\begin{array}{c}18,11449 \\
20,07 \\
0,938\end{array}$ \\
\hline $\begin{array}{l}\text { Reino } \\
\text { Unido }\end{array}$ & $\begin{array}{l}\text { Dexp (Log) } \\
\text { Dexp (Kg) } \\
\text { Dam } \\
\text { Dso }\end{array}$ & $\begin{array}{l}147 \\
147 \\
147 \\
147\end{array}$ & $\begin{array}{c}14,13904 \\
2.990 .968 \\
8,91 \\
0,858\end{array}$ & $\begin{array}{c}1,146669 \\
3.518 .074 \\
5,02 \\
0,065\end{array}$ & $\begin{array}{c}10,54289 \\
37.907 \\
-5,70 \\
0,683\end{array}$ & $\begin{array}{c}16,545 \\
15.300 .000 \\
21,67 \\
0,938\end{array}$ \\
\hline $\begin{array}{l}\text { Base } \\
\text { Consolidada }\end{array}$ & $\begin{array}{l}\text { Dexp (Log) } \\
\text { Dam } \\
\text { Dso }\end{array}$ & $\begin{array}{l}959 \\
959 \\
959\end{array}$ & $\begin{array}{c}14.81024 \\
9,32 \\
0.861\end{array}$ & $\begin{array}{c}2.242304 \\
4.35 \\
0.070\end{array}$ & $\begin{array}{c}9.805489 \\
-5.70 \\
0.549\end{array}$ & $\begin{array}{c}19,7 \\
24.17 \\
0.938\end{array}$ \\
\hline
\end{tabular}

Fonte: Dados da pesquisa.

\subsection{Resultados das estimações}

A Tabela 2 apresenta os resultados dos testes de especificação do modelo proposto. O teste de Hausman busca verificar se o método mais apropriado para realizar as estimações é um modelo de regressão de efeitos fixos ou de efeitos aleatórios, em que a não rejeição da hipótese nula indica que o modelo mais adequado é de efeitos fixos, enquanto sua 
rejeição aponta que o modelo de regressão mais adequado é de efeitos aleatórios. Conforme pode ser verificado nas informações contidas na Tabela 2, o teste de Hausman aponta que o modelo de regressão de efeitos fixos se mostra mais adequado para realizar as estimações do modelo.

Tabela 2 - Resultado dos testes de especificação do modelo.

\begin{tabular}{c|c}
\hline Teste de Especificação & Modelo Consolidado \\
\hline Teste de Hausman & $\begin{array}{c}\text { Chi2 }=21,01 \\
\mathrm{p}>\mathrm{chi} 2=0,0000\end{array}$ \\
\hline FIV & $\mathrm{FIV}=1,00$ \\
\hline Teste de BreushPagan e Godfrey & $\begin{array}{c}\text { Chi2 }=615,32 \\
\mathrm{p}>\mathrm{chi} 2=0,0000\end{array}$ \\
\hline Teste de Wooldridge & $\mathrm{F}(1,100)=127,147$ \\
$\mathrm{p}>\mathrm{F}=0,0054$
\end{tabular}

Fonte: Dados da pesquisa.

O FIV é uma medida do grau em que cada variável independente é explicada pelas demais variáveis independentes (HAIR et al., 2009). Segundo os autores, quanto maior o valor do FIV, maior o grau de multicolinearidade entre as variáveis, determinando como limite de tolerância um FIV de referência de 10, em que uma variável com esse valor é tida como altamente colinear. Dessa forma, conforme os resultados obtidos para o FIV do modelo proposto, não há indícios de multicolinearidade entre as variáveis dos modelos propostos.

A homocedasticidade se refere à suposição de que as variáveis dependentes do estudo possuem níveis iguais de variância em relação às suas variáveis independentes (HAIR et al., 2009). Para testar a homocedasticidade nos resíduos, foi feito o teste de Breush-PaganGodfrey, em que a não rejeição da hipótese nula indica que a variância dos termos de erro não são constantes entre as variáveis independentes. Tal análise apontou para a presença de heterocedasticidade no modelo.

A autocorrelação consiste na correlação entre os termos de erro de séries de observações ordenadas no tempo ou no espaço. 
Entretanto, os pressupostos de regressão linear consideram que essa autocorrelação não existe (GUJARATI; PORTER, 2011). Por isso, para testar a presença de autocorrelação nos termos de erro dos modelos propostos, foi feito o teste de Wooldridge, cuja não rejeição da hipótese nula aponta para a existência de autocorrelação. Como pode ser verificada nos resultados apresentados na Tabela 1, a análise confirmou a presença de autocorrelação nos modelo proposto.

Diante da presença de heterocedasticidade e autocorrelação nos modelos de regressão propostos, adotou-se um procedimento de correção robusta dos erros padrão, conforme indicado por Wooldridge (2013). O resultado obtido pelo modelo de regressão de efeitos fixos, aplicando uma correção robusta de erros, é reproduzido na Tabela 3.

Tabela 3 - Análise do modelo econométrico

\begin{tabular}{l|c|c|c|c|c}
\hline Modelo & $\begin{array}{c}\text { Var. Dep.: Dexp } \\
\text { (log) }\end{array}$ & Coef. & S.E. & T & p>t \\
\hline \multirow{3}{*}{ Consolidado } & Dam & -.0029013 & & & \\
& Dso & 20.95022 & 0.187675 & -0.15 & 0,877 \\
& & & 6.342875 & 3.30 & 0,001 \\
& $R^{2}$ within & 0,0558 & & & \\
& $R^{2}$ Between & 0,0136 & & & \\
\hline
\end{tabular}

Fonte: Dados da pesquisa.

Os resultados obtidos na avaliação do relacionamento entre o desempenho social e o desempenho das exportações de carne bovina in natura fresca ou resfriada apontam para a existência de uma relação positiva e significativa entre o desempenho exportador e o desempenho social, devido à estatística $T=3,30$, com um $p$ valor de 0,001 e um coeficiente positivo (20.95022), confirmando a Hipótese 1 do estudo. Por outro lado, os resultados obtidos para a análise do relacionamento entre o desempenho ambiental e o desempenho das exportações de carne bovina não dão suporte para a Hipótese 2. 


\subsection{Discussão dos resultados}

O presente trabalho avalia o relacionamento entre desempenho social e desempenho exportador a partir de uma análise em âmbito nacional, em um mercado marcado pela elevada concentração, dominado por poucas empresas multinacionais que controlam a cadeia de valor do produto. Na última década, as principais empresas da cadeia produtiva da carne bovina têm investido no aprimoramento de suas práticas de gestão, de forma a atender as demandas públicas por uma atuação responsável, e vêm reportando a evolução das suas práticas de gestão socioambiental através da publicação de relatórios de gestão que atendem a padrões aceitos internacionalmente. Além de atender a uma demanda do mercado por informações sobre processos produtivos e seu impacto sobre colaboradores e comunidades onde atuam, essas empresas estreitam suas relações com fornecedores e o mercado, cultivando uma reputação socialmente responsável, que Ihes facilita o acesso aos principais mercados e barreiras a empresas que atuam com padrões socioambientais inferiores ou não atendem às demandas por informações.

Neste estudo, identificou-se que os exportadores de carne bovina que apresentam desempenho social mais elevado também apresentam um desempenho exportador mais elevado, confirmando a Hipótese 1 do estudo. Uma possível explicação para esse resultado parte da lógica de que países com IDH mais elevado possuem melhores níveis de desenvolvimento social, força de trabalho melhor qualificada e população com nível de consciência social elevada, exigindo que firmas e governos atuem de forma sustentável. Nesse sentido, as empresas capazes de atender às demandas por um desempenho social superior nesses países podem se apropriar das vantagens obtidas nesses mercados, como ganhos de produtividade e a construção de uma reputação socialmente responsável, e transferi-las para mercados com demandas semelhantes, tornando-se capazes de atingir um desempenho superior.

Já a Hipótese 2 do estudo propõe que o desempenho ambiental está positivamente relacionado com o desempenho exportador. Nesse 
sentido, diante dos problemas ambientais decorrentes da expansão da produção pecuária nas últimas décadas, o impacto gerado pelo setor sobre o meio ambiente passou a receber cada vez mais atenção de entidades públicas e privadas. Com isso, as empresas do setor se tornaram suscetíveis ao controle regulatório de governos e organizações internacionais, danos à imagem e riscos financeiros decorrentes de penalidades, como multas e cancelamentos de contratos de fornecimento, pelo mau uso de recursos naturais pelas atividades produtivas.

Os trabalhos de Martín-Tapia, Aragón-Correa e Senize-Barrio (2008) e Antonietti e Marzucchi (2013) constatam que a adoção de processos produtivos ambientalmente sustentáveis permite um aumento da eficiência produtiva, que resulta na redução de custos operacionais com um melhor aproveitamento de matérias-primas, evitando danos à imagem corporativa, a redução de custos associados a litígios e multas decorrentes de problemas ambientais. Além disso, a adoção de processos sustentáveis confere a essas empresas acesso facilitado a mercados com normas ambientais mais rigorosas, possibilitando um aumento das receitas de venda e, consequentemente, desempenho exportador superior ao obtido por empresas com padrões ambientais inferiores.

A análise estatística para a Hipótese 2 acabou não confirmando a proposta apresentada. O setor produtivo da carne bovina é caracterizado por ser intensivo em recursos naturais e por uma forte concentração entre as indústrias exportadoras, o que gera uma busca incessante por economias de escala e baixos preços de comercialização, resultando em um impacto significativo sobre o meio ambiente. Entretanto, nas últimas décadas, frente a pressões institucionais para reduzir seu impacto sobre o meio ambiente, as empresas exportadoras de carne bovina passaram a adotar práticas de controle sanitário e rastreabilidade da produção, incentivando os produtores a adotar melhores práticas de manejo do rebanho e medidas de redução do impacto das suas atividades produtivas (MALAFAIA et al., 2010). Ao mesmo tempo, é cada vez maior a exigência de certificação de produtos agrícolas por parte de varejistas que atuam nos mercados analisados, preocupados em melhorar sua 
imagem junto a um público consumidor cada vez mais preocupado com questões de saúde, segurança alimentar, não utilização de agrotóxicos e não degradação do meio ambiente (PAULINO; SCIENCIA, 2013).

Embora a análise econométrica aponte para a inexistência de relação entre o desempenho social e o desempenho exportador, os mercados exercem uma rígida seleção de fornecedores por meio de barreiras tarifárias e sanitárias. Nesses mercados, as empresas exportadoras de carne que não obedecem a uma série de requisitos éticos, técnicos e sanitários, e comprovam que suas atividades produtivas não degradam o meio ambiente, estão passíveis de sofrer sanções econômicas e comerciais, podendo ser excluídas ou nem mesmo ter acesso a esses mercados. Nesse sentido, uma possível explicação para os resultados obtidos é que a influência do desempenho ambiental sobre o desempenho exportador pode se tornar nula diante da obrigatoriedade de os frigoríficos de carne bovina atender às exigências éticas, técnicas, sanitárias e ambientais para entrada e manutenção da posição nos mercados importadores.

Esses resultados demonstram que a dimensão social da sustentabilidade exerce uma influência positiva sobre o desempenho exportador dos frigoríficos exportadores de carne bovina, enquanto a dimensão ambiental da sustentabilidade está associada à capacidade de entrada e manutenção destas empresas no mercado internacional da carne bovina.

Ao mesmo tempo, diante da obrigatoriedade de as empresas atenderem a um padrão de atuação sustentável para ter acesso ao mercado internacional da carne bovina, institucionalizando um padrão de atuação sustentável dentro do setor, considera-se que, embora a sustentabilidade seja fundamental para a competitividade das empresas do setor, a concorrência dentro da indústria exportadora de carne bovina deve continuar a recrudescer, em razão da alta concentração do setor no mercado internacional, devendo ser enfrentada por meio da capacidade de as empresas atenderem a requisitos éticos, técnicos, sanitários e ambientais impostos pelos mercados importadores, e ainda obterem economias de escala e preços baixos de comercialização. 


\section{Conclusão}

Este estudo buscou avaliar o relacionamento entre a sustentabilidade e o desempenho exportador a partir da análise das exportações de carne bovina in natura fresca ou congelada, entre os anos 2002 e 2012, para os mercados italiano, norte-americano, holandês, francês, mexicano, japonês, alemão e para o Reino Unido, responsáveis pela compra de $70 \%$ do produto transacionado durante o período no mercado internacional.

Para atingir esse objetivo, em um primeiro momento, propôsse avaliar a influência do desempenho social sobre o desempenho exportador da indústria frigorífica. A literatura aponta que empresas que atuam com os mais altos padrões éticos e sociais são capazes de obter vantagens competitivas por meio de uma reputação socialmente sustentável, possibilitando a cobrança de valores superiores por seus produtos, ao mesmo tempo em que facilita o acesso dessas empresas a mercados mais exigentes. Nesse sentido, a Hipótese 1 do estudo propôs que o desempenho social está positivamente relacionado ao desempenho das exportações de carne in natura fresca ou resfriada.

O resultado da análise econométrica dá suporte à Hipótese 1, apontando que empresas com um desempenho social superior também apresentam um desempenho exportador superior no mercado internacional da carne bovina. Empresas capazes de atender às demandas pelo desenvolvimento de práticas socialmente responsáveis em países com desenvolvimento humano mais elevado podem se apropriar de vantagens obtidas nesses mercados, como ganhos de produtividade devido à contratação de mão de obra melhor capacitada, e transferi-las para mercados com demandas semelhantes. Ao mesmo tempo, essas empresas estreitam suas relações com fornecedores e o mercado cultivando uma reputação socialmente responsável, que Ihes facilita o acesso aos principais mercados impondo barreiras a empresas que atuam com padrões sociais inferiores no seu mercado doméstico e no mercado internacional, tornando-se capazes de atingir um desempenho superior. 
Posteriormente, foi avaliada a influência do desempenho ambiental sobre o desempenho exportador. Aadoção de processos ambientalmente sustentáveis pode resultar em reduções de custos operacionais, ao mesmo tempo em que possibilita acesso facilitado a mercados de países com normas ambientais mais rigorosas, garantindo um aumento das receitas de venda e, consequentemente, um desempenho exportador superior. Nesse caso, a proposta da Hipótese 2 reflete uma expectativa para que o desempenho ambiental influencie positivamente o desempenho exportador. Porém, a análise econométrica não suportou tal hipótese. Embora o setor produtivo da carne bovina seja intensivo em recursos naturais, nas últimas décadas, as empresas exportadoras passaram a adotar uma série de medidas que visam reduzir o impacto das suas atividades produtivas sobre o meio ambiente, visto que é recorrente a exigência, por parte dos principais varejistas, da comprovação de que as atividades produtivas não degradam o meio ambiente. Empresas que não atendem aos requisitos impostos pelos mercados consumidores correm o risco de sofrer sanções econômicas e comerciais, podendo ser excluídas ou sequer ter acesso a estes mercados. Nesse sentido, considerando os pressupostos da teoria institucional, segundo a qual pressões ambientais resultam em processos isomórficos que tornam as empresas homogêneas em um mesmo campo organizacional obrigandoas a adotar práticas comuns (DIMAGGIO; POWELL, 1983), a adoção de práticas sustentáveis tende a se tornar prática institucionalizada entre as empresas do setor.

Os resultados obtidos pela análise econométrica demonstram que a dimensão social da sustentabilidade exerce uma influencia positiva sobre o desempenho exportador dos frigoríficos exportadores de carne bovina, enquanto a dimensão ambiental da sustentabilidade está associada à capacidade de entrada e manutenção destas empresas no mercado internacional da carne bovina. Diante desses resultados, considera-se que a adoção de processos produtivos sustentáveis é requisito para entrada e manutenção das firmas no mercado internacional ao mesmo tempo em que influencia positivamente o desempenho das exportações de carne bovina in natura fresca ou resfriada. 
Como toda pesquisa, este estudo conta com limitações. A utilização de dados nacionais é uma limitação importante da pesquisa. A análise em âmbito nacional se deve à indisponibilidade de informações sobre comércio exterior em termos de firma para as empresas do setor. Porém, haja vista o nível elevado de concentração na indústria exportadora de carne e, ainda, que a cadeia de valor e o mercado internacional do produto são dominados por um pequeno grupo de grandes empresas com presença global, considera-se que, através da análise do comportamento do comércio entre países, seja possível inferir o comportamento do comércio da commodity em nível de firma. Nesse sentido, novos estudos poderão utilizar dados em nível de firma para reduzir o nível de incerteza dos resultados.

O estudo também apresenta limitações devido a não utilização de variáveis de controle, o que não permite descartar explicações alternativas para os resultados encontrados para a análise econométrica. Estudos futuros podem utilizar variáveis de controle como preço, distância entre os mercados importador e exportador, renda e indicadores de demanda de carne bovina no mercado internacional.

Os achados da pesquisa trazem importantes contribuições gerenciais uma vez que os gestores podem ponderar a localização das suas unidades produtivas, buscando áreas de produção com desenvolvimento social elevado. Nessas áreas, os frigoríficos podem se apropriar de uma força de trabalho melhor qualificada e obter ganhos de produtividade ao mesmo tempo em que podem construir uma reputação socialmente responsável, visto o nível de desenvolvimento social elevado das comunidades onde atuam. Para maximizar seu desempenho exportador, os frigoríficos exportadores devem maximizar seu desempenho social e, ao mesmo tempo, ter acesso e manter sua posição nos mercados estrangeiros. Para isso, além de atender os requisitos técnicos e sanitários impostos pelos países importadores, os frigoríficos exportadores de carne bovina devem comprovar que suas atividades produtivas não degradam o meio ambiente. Nesse sentido, os gestores podem avaliar suas práticas ambientais e a escolha de tecnologias de produção visando desenvolver estratégias de longo 
prazo com o intuito de fortalecer sua posição no mercado internacional e se antecipar ao surgimento de novas demandas por parte dos mercados importadores.

Tais resultados poderão ainda ser utilizados como instrumento de políticas públicas que disseminem a adoção de práticas produtivas sustentáveis dentro da cadeia produtiva da carne bovina, demonstrando a necessidade de uma postura sustentável para entrada e manutenção dos mercados estrangeiros, além das vantagens obtidas por empresas sustentáveis dentro do mercado internacional.

\section{Referências}

AGUINIS, H.; GOTTFREDSON, R. K.; JOO, H. Best-practice recommendations for defining, identifying, and handling outliers.

Organizational Research Methods, Thousand Oaks, CA, v. 16, n. 2, p. 270-301, Mar. 2013.

AMEER, R.; OTHMAN, R. Sustainability Practices and Corporate Financial Performance: A Study Based on the Top Global Corporations. Journal of Business Ethics, Dordrecht, v. 108, p. 61-79, June 2012.

ANDERSEN, M.; SKJOETT-LARSEN, T. Corporate social responsibility in global supply chains. Supply Chain Management: An International Journal, United Kingdom, v. 14, n. 2, p. 75-86, Mar/Apr 2009.

ANTONIETTI, R., MARZUCCHI, A. Green investment strategies and export performance: a firm-level investigation. Ecological Economics, [S.I.], v.108, p.150-161, Dec. 2014.

BANCO MUNDIAL. Adjusted Net Saving. Disponível em: <http://web. worldbank.org/wbsite/external/topics/environment/exteei/0,,contentmd k:20502388 menupk:1187778 pagepk:210058 pipk:210062 thesite pk:408050,00.html>. Acesso em: 15 jun. 2014.

. World databank. Disponível em: <http://databank.worldbank. org/data/views/variableselection/selectvariables.aspx?source=wea Ithaccounting>. Acesso em: 15 jun. 2014b. 
BELLESI, F.; LEHRER, D.; TAL, A. Comparative advantage: the impact of ISO 14001 environmental certification on exports. Environmental science \& technology, Washington, DC, v. 39, n. 7, p. 1943-1953, Apr. 2005.

BILLOR, N.; HADI, A. S.; VELLEMAN, P. F. BACON: blocked adaptive computationally efficient outlier nominators. Computational Statistics \& Data Analysis, Amsterdam, v. 34, n. 3, p. 279-298, Sept. 2000.

BOEHE, D. M.; BARIN CRUZ, L. Corporate Social Responsibility, Product Differentiation Strategy and Export Performance. Journal of Business Ethics, Dordrecht, v. 91, n. S2, p. 325-346, Feb. 2010.

BOWEN, H. R. Social responsibilities of the businessman. New York: Harper-Row, 1953.

CARNEIRO, J.; ROCHA, A.; SILVA, J. Determinants of export performance: a study of large brazilian manufacturing firms. BARBrazilian Administration Review, Rio de Janerio., v. 8, n. 2, p. 107132, Apr./Jun. 2011.

CARROLL, A. A three-dimensional conceptual model of corporate performance. Academy of management review, United States, v. 4, n. 4, p. 497-505, Oct. 1979.

CARTER, C. R.; ROGERS, D. S. A framework of sustainable supply chain management: moving toward new theory. International Journal of Physical Distribution \& Logistics Management, United Kingdo, v. 38, n. 5, p. 360-387, Jul./Aug. 2008.

CHANDLER, A. D. Strategy and structure: Chapters in the history of the industrial enterprise. Boston: MIT Press, 1969.

DIMAGGIO, P.; POWELL, W. The iron cage revisited: Institutional isomorphism and collective rationality in organizational fields.

American sociological review, Notre Dame, IN, v. 48, n. 2, p. 147160, Apr. 1983. 
DIXON-FOWLER, H. R. et al. Beyond "Does it Pay to be Green?" A Meta-Analysis of Moderators of the CEP-CFP Relationship. Journal of Business Ethics, Dordrecht, v. 112, n. 2, p. 353-366, Jan. 2012. ( doi:10.1007/s10551-012-1268-8)

\section{DYCK, J. H.; NELSON, K.E. Structure of the global markets for} meat. Trade Economics Division,Economic Research Service,U.S. Department of Agriculture. USDA Agriculture Information Bulletin, $\mathrm{n}^{\circ}$ 785, Washington D.C., 2003.

DYLLICK, T.; HOCKERTS, K. Beyond the business case for corporate susteinability. Business Strategy and the Environment, United Kingdom, v. 11, n. 2, p. 130-141, Mar./Apr. 2002.

ELKINGTON, J. Cannibals with forks: the triple bottom line of 21st century business. Oxford, Reino Unido: Capstone, 1997.

FAO. FAOSTAT Database. Disponível em: <http://faostat3.fao.org/ faostat-gateway/go/to/home/E>. Acesso em: 15 jun. 2014.

FLORES, M. Mercado mundial y cadena de valor de la carne bovina. In: Seminario Anual del Programa Grupos I+D "Cambios en la sociedad rural a inicios del siglo XXI”, 2012, Montevideo. Anais... Montevideo, UY: Universidad de La República, 2012. Disponível em: < http:// cienciassociales.edu.uy/departamentodesociologia/wp-content/ uploads/sites/3/2013/archivos/90.pdf >. Acesso em: 01 dez. 2014.

FRIEDMAN, M. The social responsibility of business Is to increase its profits. New York Times Magazine, Nova lorque, v. 13, p. 32-33, Sept. 1970.

GALDEANO-GÓMEZ, E. Exporting and environmental performance: firm-level productivity analysis. World Economy, Oxford, v. 33, n. 1, p. 60-88, Jan. 2010.

GAVRONSKI, I.; FERRER, G.; PAIVA, E. L. ISO 14001 certification in Brazil: motivations and benefits. Journal of Cleaner Production, Amsterdam, v. 16, n. 1, p. 87-94, Jan. 2008. 
GUJARATI, D.N.; PORTER, D. C. Econometria Básica. 5 ed. Porto Alegre: AMGH, 2011. P. 924.

HAIR, Joseph F. et al. Análise Multivariada de Dados. 6 ed. Porto Alegre: Bookman, 2009. P. 688.

HART, S. L. A Natural-Resource-Based View of the Firm. Academy of Management Review, United States, v. 20, n. 4, p. 986, Oct. 1995.

HULL, C. E.; ROTHENBERG, S. Firm performance: the interactions of corporate social performance with innovation and industry differentiation. Strategic Management Journal, Sussex, England, v. 29, n. 7, p. 781-789, Feb 2008.

INTERNATIONAL ORGANIZATION FOR STANDARDIZATION. ISO International Standards. Disponìvel em:<http://www.iso.org>. Acesso em: 10 out. 2014 .

KING, A.; LENOX, M. Exploring the locus of profitable pollution reduction exploring the locus of profitable pollution reduction.

Management Science, Philadelphia, v. 48, n. 2, p. 289-299, Feb. 2002.

LAWRENCE, P. R.; LORSCH, J. W. Organization and environment: Managing differentiation and integration. Boston : Division of Research, Graduate School of Business Administration: Harvard University, 1967.

LEE, K.-H.; KIM, J.-W. Current status of CSR in the realm of supply management: the case of the Korean electronics industry. Supply Chain Management: An International Journal, United Kingdom, v. 14, n. 2, p. 138-148, Mar./Apr. 2009.

LEONIDOU, L. C. et. al. Antecedents and consequences of an ecofriendly export marketing strategy : the moderating role of competitive intensity. Journal of International Marketing, Chicago, v. 21, n. 3, p. 22-46, Sept. 2013.

LEONIDOU, L. C.; KATSIKEAS, C. S.; PIERCY, N. F. Identifying managerial influences on exporting: past research and future 
directions. Journal of International Management, United States, v. 6, n. 2, p. 74-102, Apr./Jun. 1998.

MALAFAIA, G. C., BARCELLOS, J. O. J.; PEDROZO, E. A.; CAMARGO, M.E. Convention economics and coordinhation mechanisms in collective actions: The Uruguay certified beef case. Journal of Development and Agricultural Economics. Nairobi, v. 2, n. 3, p. 178-187, May 2010.

MARTINS, C. H. B.; OLIVEIRA, N. Indicadores EconômicoAmbientais na Perspectiva da Sustentabilidade. Documentos FEE; n. 63. Porto Alegre: FEE -FEPAM, 2005.

MARTÍN-TAPIA, I.; ARAGÓN-CORREA, J. A.; RUEDA-MANZANARES, A. Environmental strategy and exports in medium, small and microenterprises. Journal of World Business, United Kingdom, v. 45, n. 3, p. 266-275, July 2010.

MARTÍN-TAPIA, I.; ARAGÓN-CORREA, J. A.; SENISE-BARRIO, M. E. Being green and export intensity of SMEs: The moderating influence of perceived uncertainty. Ecological Economics, Amsterdam, v. 68, n. 1-2, p. 56-67, dez.. 2008.

MATUTE, J.; FRAJ, E.; MARTI, E. Green marketing strategy and the firm's performance : the moderating role of environmental culture. Journal of Strategic Marketing, United Kingdom, v. 19, n. 4, p. 339355, July 2011.

MORGAN, N. A.; KALEKA, A.; KATSIKEAS, C. S. Antecedents of export venture performance: a theoretical model and empirical assessment. Journal of Marketing, Chicago, IL v. 68, n. 1, p. 90-108, Jan. 2004.

UN COMTRADE DATABASE. United Nations Commodity Trade Statisticas Database - COMTRADE. Disponível em: <http://comtrade. un.org>. Acesso em: 15 jun 2014. 
UNITED NATIONS STATISTICS DIVISION. Undata a world of information. Disponível em: <http://data.un.org>. Acesso em: 15 June 2014b.

ORLITZKY, M.; SIEGEL, D. S.; WALDMAN, D. A. Strategic corporate social responsibility and environmental sustainability. Business \& Society, Chicago, v. 50, n. 1, p. 6-27, Jan. 2011.

PALMA, E. P., GOMES, C.M., KNEIPP, j.m., rosa, I.a.b. estratégias de Negócios Sustentáveis e Desempenho Exportador: uma análise em empresas do setor de gemas e joias. Revista Brasileira de Gestão de Negócios, São Paulo, v. 16, n. 50, p. 25-42 Jan./Mar. 2014.

PAULINO, S. R.; SCIENCIA, I. Impactos ambientais da certificação globalgap em agroindústrias de alimentos. In: Congresso da Sociedade Brasileira de Economia, Administração e Sociologia Rural, 48, 2013, Campo Grande-MS. Anais... Campo Grande-MS: CSBEAS, 2013. Disponível em: <http://www.sober.org.br/palestra/15/118.pdf>. Acesso em: 10 Mar. 2015.

PORTER, M. E.; KRAMER, M. K. Strategy and society: the link between competitive advantage and corporate social reponsibility. Harvard Business Review, Boston, v. 85, n. 12, Dec. 2006.

PORTER, M. E.; LINDE, C. V. D. Toward a new conception of the environment-competitiveness relationship. The Journal of Economic Perspectives, Pittsburgh, v. 9, n. 4 (Autumn), p. 97-118, 1995.

PRAHALAD, C.; HAMEL, G. The core competence of the corporation. Harvard Business Review, Boston. v. 68, n. 3, p. 79-90, May.-June, 1990.

RODRIGUEZ-RODRIGUEZ, M., GALDEANO-GÓMEZ, E., CARMONAMORENO, E., GODOY-DURAN, A. Environmental Impact, Export Intensity, and Productivity Interactions: An Empirical Index Analysis of the Agri-Food Industry in Spain. Canadian Journal of Agricultural Economics, Canada, v. 60, n. 1, p. 33-52. Mar. 2012. 
RUSSO, M. V.; FOUTS, P.A.. A Resource-Based perspective on corporate environmental performance and profitability'. Academy of Management Journal, Nova lorque, v. 40 n. 3, p. 534-559, Jun. 1997.

SCHLESINGER, S. Onde pastar? O gado bovino no Brasil. Rio de Janeiro: FASE, 2010.

SOUSA, C. M. P.; MARTÍNEZ-LÓPEZ, F. J.; COELHO, F. The determinants of export performance: A review of the research in the literature between 1998 and 2005. International Journal of Management Reviews, Londres v. 10, n. 4, p. 343-374, Nov. 2008.

STATACORP. Stata Statistical Software: Release 13. College Station, TX: StataCorp LP. 2013.

STEHFEST, E. et. al. Options to reduce the environmental effects of livestock production - Comparison of two economic models.

Agricultural Systems, England, v. 114, p. 38-53, Jan. 2013.

UNITED STATES DEPARTMENT OF AGRICULTURE (USDA).

Production, supply and distribution online. Disponível em: <http:// apps.fas.usda.gov/psdonline/psdQuery.aspx>. Acesso em: 15 jun. 2014.

VIEIRA, L. M., TRAILL, W. B. Trust and governance of global value chains. British Food Journal, Surrey, England, v. 110, n. 4/5, p. 460473, Apr./May. 2008.

WHEELER, D. Racing to the bottom? Foreign investment and air pollution in developing countries. The Journal of Environment \& Development, New York, v. 10, n. 3, p. 225-245, Jan. 2001.

WOOLDRIDGE, J. M. Introdução à econometria: uma abordagem moderna. 4. ed. São Paulo: Cengage Learning, 2013. 701p.

WORLD COMMISSION on Environment and Development (WCDE). Our common future. Oxford: Oxford University Press, 1987. 
ZEN, S. et.al. Pecuária de corte brasileira: impactos ambientais e emissões de gases efeito estufa (GEE). Piracicaba-SP: Esalq/Cepea, 2008.

ZOU, S.; TAYLOR, C. R.; OSLAND, G. E. The EXPERF scale : a cross-national generalized export performance measure. Journal of International Marketing, Chicago, v. 6, n. 3, p. 10, Jan. 1998.

Artigo recebido em: 29/10/2015

Aprovado em: 01/02/2016 